Bull. Austral. Math. Soc.

$60 \mathrm{~J} 65,31 \mathrm{~B} 15,31 \mathrm{~A} 15$

Vol. 66 (2002) [233-244]

\title{
HITTING PROBABILITIES OF CONDITIONAL BROWNIAN MOTION AND POLARISATION
}

\author{
Dimitrios Betsakos
}

\begin{abstract}
We study the behaviour of the hitting probabilities of conditional Brownian motion in a domain $D$ in Euclidean space when we apply polarization to $D$. We also study how polarization affects the probability that conditional Brownian motion meets a subset of $D$.
\end{abstract}

\section{INTRODUCTION}

In 1957, Doob [8] defined the conditional Brownian motion. A comprehensive study of this Markov process from the point of view of classical potential theory is contained in Doob's treatise [9]. In this paper we study some geometric properties of conditional Brownian motion in $\mathbb{R}^{n}, n \geqslant 2$.

Let $D$ be a Greenian domain in $\mathbb{R}^{n}$, and let $h$ be a strictly positive superharmonic function in $D$. Brownian motion in $D$ conditioned by $h$ (or briefly $h$-Brownian motion) is a Markov process with state space $D$ and transition density

$$
p_{D}^{h}(t, \xi, \eta)=\frac{1}{h(\xi)} p_{D}(t, \xi, \eta) h(\eta)
$$

where $p_{D}(t, \xi, \eta)$ is the transition density of the (usual, unconditioned) Brownian motion killed on exiting $D$. Two important special cases for $h$ are the following:

(a) Let $\zeta$ be a point on the Martin boundary of $D$ and let $h=K_{D}(\zeta,:)$, where $K_{D}(\zeta, \cdot)$ is the Martin kernel function of $D$ with pole at $\zeta$. Then $h$-Brownian motion is conditioned to exit $D$ at the given point $\zeta$.

(b) Let $\xi \in D$ and let $h=G_{D}(\xi, \cdot)$ be the Green function of $D$ with pole at $\xi$. Then $h$-Brownian motion is conditioned to hit the point $\xi$ before leaving D.

All conditional Brownian motions are mixtures of these two basic processes. Let $\mathbb{P}_{\xi}^{h}$ denote the measure (on the path space) induced by the transition density (1.1) and let $\mathbb{E}_{\xi}^{h}$ be the corresponding expectation. If $h \equiv 1$, we shall omit the superscript $h$.

Received 24th January, 2002

Copyright Clearance Centre, Inc. Serial-fee code: 0004-9727/02 \$A2.00+0.00. 
In Section 3 we study the behaviour of hitting probabilities ("conditional harmonic measures") of $h$-Brownian motion under the geometric transformation called polarisation. The definition and main properties of polarisation are reviewed in Section 2. In Section 4 we study the probability of meeting a set in relation with polarisation and in Section 5 we give as application a symmetrisation result that extends a classical theorem of A.Baernstein. In general, the geometric behaviour of conditional Brownian motion is not well-understood. Some concluding remarks, open problems and conjectures appear in Section 6.

\section{Polarisation}

Polarisation was introduced by Wolontis [16] in 1952. Before stating its definition we need to introduce some notation: Let $\mathbb{H}$ be the $(n-1)$-dimensional plane $\left\{\left(x_{1}, x_{2}, \ldots, x_{n}\right) \in \mathbb{R}^{n}: x_{n}=0\right\}$ and $\mathbb{R}_{+}^{n}$ be the half-space $\left\{\left(x_{1}, x_{2}, \ldots, x_{n}\right) \in \mathbb{R}^{n}: x_{n}\right.$ $>0\}$. Let "hat" denote reflection in $\mathbb{H}$; that is, if $x=\left(x_{1}, x_{2}, \ldots, x_{n}\right) \in \mathbb{R}^{n}$ and $A \subset \mathbb{R}^{n}$ then $\widehat{x}:=\left(x_{1}, x_{2}, \ldots,-x_{n}\right)$ and $\widehat{A}:=\left\{x \in \mathbb{R}^{n}: \widehat{x} \in A\right\}$.

Polarisation is defined as follows: Let $A$ be a set in $\mathbb{R}^{n}$. We divide it into three subsets $A_{1}, A_{2}, A_{3}$ :

$$
\begin{aligned}
& A_{1}=\{x \in A: \widehat{x} \in A\}=A \cap \widehat{A} \text { (the "symmetric part" of } A \text { ), } \\
& A_{2}=\left\{x \in A: x \in \mathbb{R}_{+}^{n} \text { and } \widehat{x} \notin A\right\} \text { (the "upper non-symmetric part" of } A \text { ), } \\
& A_{3}=\left\{x \in A: x \in \mathbb{R}_{+}^{n} \text { and } \widehat{x} \notin A\right\} \text { (the "lower non-symmetric part" of } A \text { ). }
\end{aligned}
$$

Then $A=A_{1} \cup A_{2} \cup A_{3}$. The polarisation $A^{*}$ of $A$ with respect to $\mathbb{R}_{+}^{n}$ is the set

$$
A^{*}:=A_{1} \cup A_{2} \cup \widehat{A_{3}} \text {. }
$$
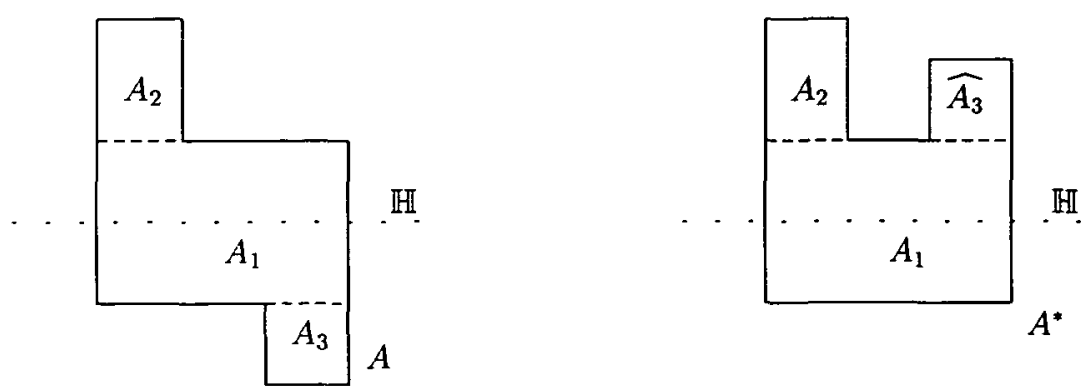

Figure 1: A set $A$ in the plane and its polarisation $A^{*}$.

We shall also use the notation: If $x=\left(x_{1}, \ldots, x_{n}\right) \in \mathbb{R}^{n}$ then $x^{*}:=\left(x_{1}, \ldots,\left|x_{n}\right|\right)$. Similarly we define polarisation with respect to other half-spaces in $\mathbb{R}^{n}$. 
By an ingenious method due to Wolontis, various types of symmetrisation can be approximated by a sequence of polarisations with respect to suitable half-spaces. Wolontis worked in the plane only but Dubinin [10] extended the method to higher dimensions. We refer to $[6,7,10,11,15]$ for some applications of this method in complex function theory, potential theory, differential equations, and Brownian motion.

In the sequel we shall use a theorem that describes the behaviour of harmonic measure under polarisation. Let $D$ be a domain in $\mathbb{R}^{n}$, regular for the Dirichlet problem, and let $B$ be a Borel set on the boundary $\partial D$ of $D$. The harmonic measure of $B$ with respect to $D$ is the solution of the Dirichlet problem in $D$ with boundary function the characteristic (indicator) function of $B$. This solution is obtained by the PWB method (see for example, [9, Chapter 1.VIII]). The value of the harmonic measure of $B$ with respect to $D$ at the point $\xi \in D$ will be denoted by $\mu_{D}(\xi, B)$. For fixed $\xi$, the set function $\mu_{D}(\xi, \cdot)$ is a probability measure on $\partial D$. In fact, $\mu_{D}(\xi, B)$ is equal to the probability that a Brownian motion in $D$ starting from $\xi$ leaves $D$ through $B$ (see [9, Chapter 2.X]). We shall say that two sets $B_{1}, B_{2}$ in $\mathbb{R}^{n}$ are equal nearly everywhere and write $B_{1} \stackrel{\text { n.e. }}{=} B_{2}$ if the set $\left(B_{1} \backslash B_{2}\right) \cup\left(B_{2} \backslash B_{1}\right)$ has zero (Newtonian) capacity. The following theorem was proved in [6] and [15]. Here we state it in a slightly improved form.

THEOREM 2.1. Let $D, B$ be as above and assume that $\widehat{B} \cap D=\emptyset$. Then

$$
\mu_{D}(\xi, B) \leqslant \mu_{D^{*}}\left(\xi, B^{*}\right), \quad \xi \in \mathbb{H} \cap D .
$$

Assume, in addition, that $D$ is not symmetric (that is, $D \neq \widehat{D}$ ). Then equality holds in (2.1) for some $\xi \in \mathbb{H} \cap D$ if and only if either $B \stackrel{\text { n.e. }}{=} B^{*}, D=D^{*}$ or $B \stackrel{\text { n.e. }}{=} \widehat{B^{*}}, D=\widehat{D^{*}}$.

\section{CONDITIONAL HARMONIC MEASURE AND POLARISATION}

Let $\Omega$ be a Greenian domain in $\mathbb{R}^{n}$. For $\xi \in \Omega$ and $h$ a positive superharmonic function in $\Omega$, we denote by $w_{\xi}^{h}$ an $h$-Brownian motion in $\Omega$ starting at $\xi$. Suppose $D$ is another domain with $D \subset \Omega$. The hitting distribution of $w_{\xi}^{h}$ on $\partial D$ is exactly the $h$-harmonic measure or conditional harmonic measure $\mu_{D}^{h}(\xi, \cdot)$; that is, the probability that an $h$-path starting from $\xi$ exits $D$ through a Borel set $B \subset \partial D$ is equal to $\mu_{D}^{h}(\xi, B)$, the latter being defined by the PWB method. The conditional harmonic measure is connected with the usual harmonic measure via the formula [9, Section 2.X.7, Section 3.II.2]

$$
\mu_{D}^{h}(\xi, B)=\frac{1}{h(\xi)} \int_{B} h(b) \mu_{D}(\xi, d b) .
$$

We shall assume from now on that the boundary of $\Omega$ consists of a finite number of smooth surfaces. In this case the Martin boundary of $\Omega$ coincides with the Euclidean boundary $\partial \Omega$. This assumption is by no means necessary but we do it in order to avoid technicalities which would only obscure the ideas we want to present in this paper. We 
shall also assume that $D^{*} \subset \Omega$. To study the behavior of $h$-harmonic measure under polarisation of $D$ we need to define a new positive superharmonic function $h^{*}$ in $\Omega^{*}$ : Let

$$
h(\xi)=v(\xi)+\int_{\Omega} G_{\Omega}(\zeta, \xi) M_{h}(d \zeta)
$$

be the Riesz decomposition of $h$; that is, $v$ is nonnegative harmonic function in $D$ and the measure $M_{h}$ is the Riesz mass of $h$ [9, Section 1.IV.8]. Let $\Omega_{h}:=\{\xi \in \Omega: v(\xi)>0\}$. For $\xi \in \Omega_{h}$, let

$$
v(\xi)=\int_{\partial \Omega} K_{\Omega}(\eta, \xi) M_{v}(d \eta)
$$

be the Martin representation of $v$ [9, Section 1.XII.9]. Here $M_{v}$ is the Martin measure for $v$ associated to $K_{\Omega}$. We consider a Borel measure $M_{h}^{*}$ on $\Omega^{*}$ such that

$$
M_{h}^{*}(A)= \begin{cases}\max \left\{M_{h}(A), M_{h}(\widehat{A})\right\}, & \text { if } A \subset \Omega^{*} \cap\left\{\left(x_{1}, \ldots, x_{n}\right): x_{n} \geqslant 0\right\} ; \\ \min \left\{M_{h}(A), M_{h}(\widehat{A})\right\}, & \text { if } A \subset \Omega^{*} \cap\left\{\left(x_{1}, \ldots, x_{n}\right): x_{n}<0\right\} .\end{cases}
$$

We assume the existence of such a measure. For example, if $M_{h}$ is absolutely continuous with respect to the $n$-dimensional Lebesgue measure then such a $M_{h}^{*}$ can be obtained by polarising the density function of $M_{h}$; or, if $M_{h}$ is discrete we can define

$$
M_{h}^{*}(\{\xi\})= \begin{cases}\max \left\{M_{h}(\{\xi\}), M_{h}(\{\widehat{\xi}\})\right\}, & \text { if } \xi=\xi^{*} \\ \min \left\{M_{h}(\{\xi\}), M_{h}(\{\widehat{\xi}\})\right\}, & \text { if } \xi=\hat{\xi}^{*}\end{cases}
$$

Similarly we consider a Borel measure $M_{v}^{*}$ on $\partial \Omega^{*}$.

We define

$$
h^{*}(\xi):=\int_{\partial \Omega} K_{\Omega}(\eta, \xi) M_{v}^{*}(d \eta)+\int_{\Omega} G_{\Omega}(\zeta, \xi) M_{h}^{*}(d \zeta), \quad \xi \in \Omega_{h}^{*} .
$$

We shall also use the notation $\Omega_{+}:=\Omega \cap \mathbb{R}^{n}, \Omega_{-}:=\Omega \cap \widehat{\mathbb{R}^{n}+}$, and the notation $\widehat{h}(x):=h(\widehat{x}), \widehat{h^{*}}(x):=h^{*}(\widehat{x})$.

THEOREM 3.1. Let $\mathbb{H}, \Omega, D, h, h^{*}$ be as above and let $B$ be a Borel set on $\partial D$. Assume that $\Omega$ is symmetric (that is, $\Omega=\widehat{\Omega}$ ) and that $\widehat{B} \cap D=\emptyset$. Then

$$
\mu_{D}^{h}(\xi, B) \leqslant \mu_{D^{*}}^{h^{*}}\left(\xi, B^{*}\right), \quad \xi \in D \cap \mathbb{H} .
$$

Assume, in addition, that $D$ is not symmetric (that is, $D \neq \widehat{D}$ ). Then equality holds in (3.2) for some $\xi \in D \cap \mathbb{H}$ if and only if either $B \stackrel{\text { n.e. }}{=} B^{*}, D=D^{*}, h=h^{*}$ or $B \stackrel{\text { n.e. }}{=} \widehat{B^{*}}, D=\widehat{D^{*}}, h=\widehat{h^{*}}$. 

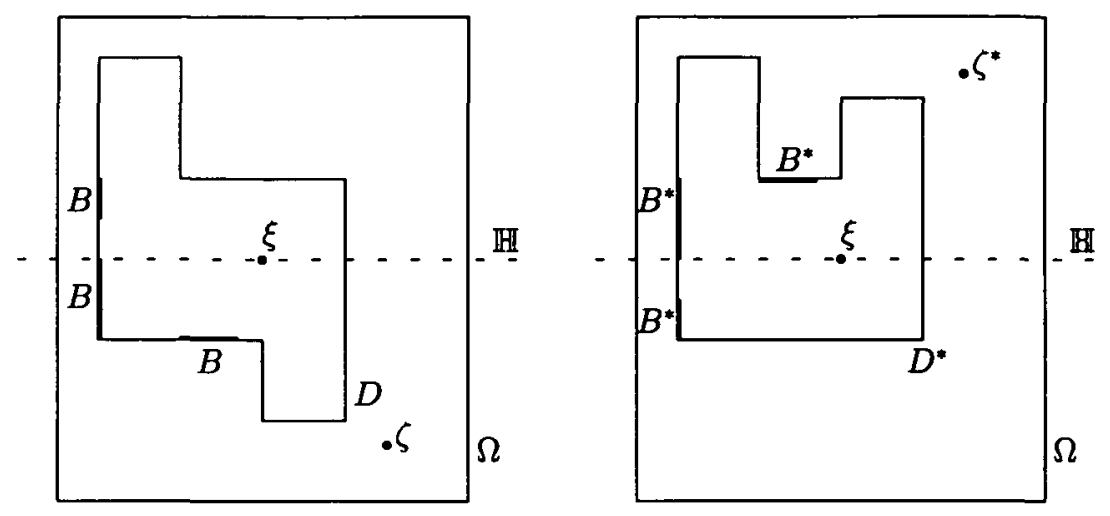

Figure 2: An illustration for Theorem 3.1.

Proof: By the construction of $h^{*}$ from $h$ and by the structure of conditional Brownian motion [9, Section 3.III.1], it suffices to consider the two basic cases: $h=G_{\Omega}(\zeta, \cdot)$ and $h=K_{\Omega}(\zeta, \cdot)$ and prove the inequalities

$$
\begin{aligned}
\int_{B} G_{\Omega}(b, \widehat{\zeta}) \mu_{D}(\xi, d b) & \leqslant \int_{B^{*}} G_{\Omega}\left(b^{*}, \widehat{\zeta}\right) \mu_{D^{*}}\left(\xi, d b^{*}\right), \quad \zeta \in \Omega_{+}, \\
\int_{B} G_{\Omega}(b, \zeta) \mu_{D}(\xi, d b) & \leqslant \int_{B^{*}} G_{\Omega}\left(b^{*}, \widehat{\zeta}\right) \mu_{D^{*}}\left(\xi, d b^{*}\right), \quad \zeta \in \Omega_{+}, \\
\int_{B}\left[G_{\Omega}(b, \zeta)+G_{\Omega}(b, \widehat{\zeta})\right] \mu_{D}(\xi, d b) & \leqslant \int_{B}\left[G_{\Omega}\left(b^{*}, \zeta\right)+G_{\Omega}\left(b^{*}, \widehat{\zeta}\right)\right] \mu_{D^{*}}\left(\xi, d b^{*}\right), \zeta \in \Omega,
\end{aligned}
$$

and the corresponding inequalities with $K_{\Omega}$ in the place of $G_{\Omega}$. We shall prove (3.3), (3.4), (3.5). The proofs of the inequalities for $K_{\Omega}$ are very similar.

The inequality (3.3) follows at once from the inequality $G_{\Omega}(b, \widehat{\zeta}) \leqslant G_{\Omega}\left(b^{*}, \zeta\right), b$ $\in B, \zeta \in \Omega_{+}$(which comes from the symmetry of $\Omega$ ) and from the inequality

$$
\mu_{D}\left(\xi, B_{1}\right) \leqslant \mu_{D^{*}}\left(\xi, B_{1}^{*}\right), \quad B_{1} \subset B
$$

which comes from Theorem 2.1.

The proof of (3.4) is similar. For the proof of (3.5) we similarly use the symmetry identity

$$
G_{\Omega}(b, \zeta)+G_{\Omega}(b, \widehat{\zeta})=G_{\Omega}\left(b^{*}, \zeta\right)+G_{\Omega}\left(b^{*}, \widehat{\zeta}\right)
$$

and the inequality (3.6).

Now we deal with the equality statement. It is clear that if $B \stackrel{\text { n.e. }}{=} B^{*}, D=D^{*}, h=$ $h^{*}$ or if $B \stackrel{\text { n.e. }}{=} \widehat{B^{*}}, D=\widehat{D^{*}}, h=\widehat{h^{*}}$, then equality holds in (3.2). Suppose, conversely, that equality holds in (3.2). 
CASE 1. $h=\widehat{h}$ (and hence $h=h^{*}=\widehat{h}^{*}$ ).

Then the inequality (3.2) comes from (3.5) and therefore if we have equality in (3.2) then we have equality in (3.5) for some $\zeta \in \Omega$. Hence we have

$$
\mu_{D}\left(\xi, B_{1}\right)=\mu_{D^{*}}\left(\xi, B_{1}^{*}\right)
$$

for all subsets $B_{1}$ of $B$ that have positive capacity. By the equality statement of Theorem 2.1 we conclude that either $B \stackrel{\text { n.e. }}{=} B^{*}, D=D^{*}$ or $B \stackrel{\text { n.e. }}{=} \widehat{B^{*}}, D=\widehat{D^{*}}$.

CASE 2. $h=h^{*}, h \neq \hat{h}$.

In this case (3.2) comes from (3.4). Equality in (3.4) for some $\zeta \in \Omega_{+}$implies (3.8). So, as in Case 1, either $B \stackrel{\text { n.e. }}{=} B^{*}, D=D^{*}$ or $B \stackrel{\text { n.e. }}{=} \widehat{B^{*}}, D=\widehat{D}^{*}$. Moreover, equality in (3.4) implies $G_{\Omega}(b, \zeta)=G_{\Omega}\left(b^{*}, \zeta\right)$ for some $\zeta \in \Omega_{+}$and nearly all $b \in B$. By the symmetry of $\Omega$, we conclude that $B \stackrel{\text { n.e. }}{=} B^{*}$. So, in Case 2 we have $B \stackrel{\text { n.e. }}{=} B^{*}, D=D^{*}$.

CASE 3. $h=\widehat{h^{*}}, h \neq \widehat{h}$.

This case is similar to Case 2. We use (3.3) and conclude that equality in (3.2) implies $B \stackrel{\text { n.e. }}{=} \widehat{B^{*}}, D=\widehat{D^{*}}$.

CASE 4. $h \neq h^{*}, h \neq \widehat{h^{*}}$.

We shall show that this case cannot occur. If $h \neq h^{*}, h \neq \widehat{h}^{*}$, then there exist $\zeta_{1}, \zeta_{2} \in \Omega_{+}$such that (3.3) (with $\zeta=\zeta_{1}$ ) holds with equality and (3.4) (with $\zeta=\zeta_{2}$ ) holds with equality. Then, as in cases 2 and 3 , we conclude that $D=D^{*}$ and $D=\widehat{D}^{*}$ which implies that $D=\widehat{D}$; contradiction.

REMARK 1. In the case where the measures $M_{h}, M_{v}$ are symmetric (that is, $M_{h}(A)$ $=M_{h}(\widehat{A}), A \subset \Omega \cap \widehat{\Omega}$ and $\left.M_{v}(A)=M_{v}(\widehat{A}), A \subset \partial \Omega \cap \partial \widehat{\Omega}\right)$, we can relax the assumption $\Omega=\widehat{\Omega}$ and assume only that $\Omega=\Omega^{*}$. Indeed, in this case, to prove (3.2), it suffices to prove (3.5) and this holds even if $\Omega=\Omega^{*} \neq \widehat{\Omega}$. Indeed, if $\Omega=\Omega^{*}$ then (3.7) holds with " $\leqslant$ " instead of "=", and this inequality together with (3.6) imply (3.5).

REMARK 2. By the same method, one can prove similar results with conditional transition densities in the place of conditional harmonic measure and/or symmetrisation in the place of polarisation.

\section{ThE PROBABILITY OF MEeting A SET}

Let $\Omega$ be a Greenian domain in $\mathbb{R}^{n}$ and $\xi, \zeta \in \Omega \cup \partial \Omega$. By $u_{\Omega}(\xi, \zeta, A)$ we denote the probability that a Brownian motion in $\Omega$ starting at $\xi$ and conditioned to go to $\zeta$ passes through a point of a compact subset $A$ of $\Omega$.

Theorem 4.1. Let $\Omega$ be a Greenian domain in $\mathbb{R}^{n}$ and $A$ be a compact subset of $\Omega$. Assume that $\Omega=\Omega^{*}$. Then we have:

(i) For $\xi, \zeta \in \mathbb{H} \cap(\Omega \cup \partial \Omega) \backslash A$,

$$
u_{\Omega}(\xi, \zeta, A) \geqslant u_{\Omega}\left(\xi, \zeta, \widehat{A^{*}}\right) .
$$


(ii) For $\xi \in \mathbb{H} \cap(\Omega \cup \partial \Omega) \backslash A$ and $\zeta \in \Omega \cup \partial \Omega \backslash A$,

$$
u_{\Omega}(\xi, \zeta, A) \geqslant u_{\Omega}\left(\xi, \zeta^{*}, \widehat{A^{*}}\right) .
$$

(iii) For $\xi, \zeta \in \Omega \cup \partial \Omega \backslash A$,

$$
u_{\Omega}(\xi, \zeta, A) \geqslant u_{\Omega}\left(\xi^{*}, \zeta^{*}, \widehat{A^{*}}\right) .
$$

(iv) For $\xi \in \mathbb{H} \cap(\Omega \cup \partial \Omega) \backslash A$ and $\zeta \in \Omega \cup \partial \Omega \backslash A$,

$$
u_{\Omega}(\xi, \zeta, A)+u_{\Omega}(\xi, \widehat{\zeta}, A) \geqslant u_{\Omega}\left(\xi, \zeta, \widehat{A^{*}}\right)+u_{\Omega}\left(\xi, \widehat{\zeta}, \widehat{A^{*}}\right)
$$
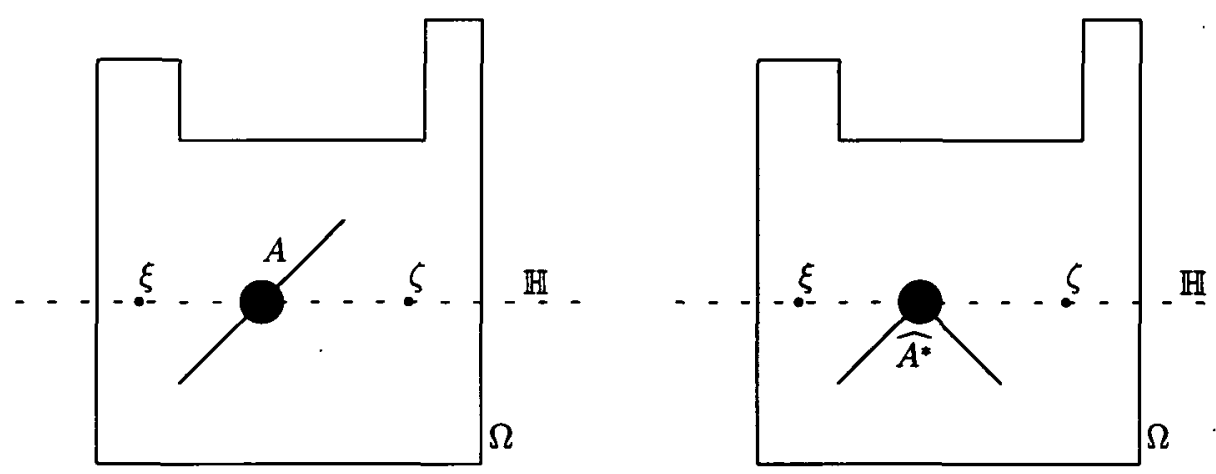

Figure 3: An illustation for Theorem 4.1.

Proof: We assume first that $\xi, \zeta \in \Omega$. We shall prove (4.1) and then show how it implies the other three inequalities via the maximum principle. Let $\delta$ be a small positive number such that the closure of the disk $\Delta=\Delta(\delta)$ with centre $\zeta$ and radius $\delta$ lies in $\Omega \backslash A$. Let $D_{\delta}:=\Omega \backslash(A \cup \Delta)$ and $h:=G_{\Omega}(\zeta, \cdot)$. By Remark 1 of the previous section, we have

$$
\mu_{D_{\delta}}^{h}(\xi, \Delta) \leqslant \mu_{D_{\xi}^{*}}^{h}(\xi, \Delta)
$$

We shall show that

$$
\lim _{\delta \rightarrow 0} \mu_{D_{\delta}}^{h}(\xi, \Delta)=1-u_{\Omega}(\xi, \zeta, A)
$$

and

$$
\lim _{\delta \rightarrow 0} \mu_{D_{\delta}^{*}}^{h}(\xi, \Delta)=1-u_{\Omega}\left(\xi, \zeta, \widehat{A^{*}}\right)
$$


For an $h$-path $\omega$ starting from $\xi$, one of the following three pairwise disjoint events must happen:

$$
\begin{aligned}
\mathcal{A}_{\delta} & :=\{\omega \text { meets } A \text { before hitting } \Delta\} \\
\mathcal{B}_{\delta} & :=\{\omega \text { meets } A \text { after hitting } \Delta\} \\
\mathcal{C} & :=\{\omega \text { does not meet } A\}
\end{aligned}
$$

Hence we have

$$
\mathbb{P}_{\xi}^{h}\left(\mathcal{A}_{\delta}\right)+\mathbb{P}_{\xi}^{h}\left(\mathcal{B}_{\delta}\right)+\mathbb{P}_{\xi}^{h}(\mathcal{C})=1
$$

It is clear that

$$
\mathbb{P}_{\xi}^{h}\left(\mathcal{A}_{\delta}\right)=1-\mu_{D_{\delta}}^{h}(\xi, \Delta)
$$

and

$$
\mathbb{P}_{\xi}^{h}(\mathcal{C})=1-u_{\Omega}(\xi, \zeta, A)
$$

By (4.8), (4.9), (4.10), to prove (4.6), it suffices to show that

$$
\lim _{\delta \rightarrow 0} \mathbb{P}_{\xi}^{h}\left(\mathcal{B}_{\delta}\right)=0
$$

By the strong Markov property and by [8, Section 13],

$$
\mathbb{P}_{\xi}^{h}\left(\mathcal{B}_{\delta}\right) \leqslant \max _{y \in \partial \Delta} u_{\Omega}(y, \zeta, A)=\max _{y \in \partial \Delta} \frac{U_{\Omega}(y, \zeta, A)}{G_{\Omega}(y, \zeta)}
$$

where $U_{\Omega}(y, \zeta, A)$ is the value at $y$ of the potential of the mass obtained by sweeping the unit mass at $\zeta$ onto $A$. Since $U_{\Omega}(\cdot, \zeta, A)$ is a continuous function on $\Omega \backslash A$ and $\zeta \notin A$, we have that for all $y$ in a neighbourhood of $\zeta$

$$
U_{\Omega}(y, \zeta, A) \leqslant M
$$

where $M$ is a positive constant not depending on $\delta$. Now (4.12), (4.13) yield (for some $y \in \partial \Delta)$

$$
\mathbb{P}_{\xi}^{h}\left(\mathcal{B}_{\delta}\right) \leqslant \frac{M}{G_{\Omega}(y, \delta)}
$$

If $\delta \rightarrow 0$, then $y \rightarrow \zeta$ and therefore $G_{\Omega}(y, \zeta) \rightarrow \infty$. Hence (4.14) implies (4.11). So we proved (4.6) and the proof of (4.7) is similar. Now (4.5), (4.6), (4.7) yield (4.1). To prove (4.2), we assume first that $\zeta \in \Omega_{+}$. Then we have to prove

$$
u_{\Omega}(\xi, \zeta, A) \geqslant u_{\Omega}\left(\xi, \zeta, \widehat{A^{*}}\right)
$$


The functions $u_{\Omega}(\xi, \cdot, A)$ and $u_{\Omega}\left(\xi, \cdot, \widehat{A^{*}}\right)$ are both $G_{\Omega}(\xi, \cdot)$-harmonic on $\Omega_{+}$and by (4.1), the inequality (4.15) holds for all $\zeta \in \partial \Omega_{+}$. So (4.15) holds for all $\zeta \in \Omega_{+}$because of the maximum principle for relative harmonic functions [9, Section 1.VIII.1]. For $\zeta \in \Omega_{-}$, (4.2) becomes

$$
u_{\Omega}(\xi, \zeta, A) \geqslant u_{\Omega}\left(\xi, \widehat{\zeta}, \widehat{A^{*}}\right)
$$

and its proof is similar to the proof of (4.15). The proofs of (4.3) and (4.4) are also similar.

So far we proved the inequalities (4.1)-(4.4) under the assumption that $\xi, \zeta \in \Omega$. If $\xi, \zeta \in \partial \Omega$ the inequalities follow from standard approximation arguments [8, Section $13]$.

\section{Application to SYMmetrisation}

By applying the approximation method of Wolontis as extended to $n$ dimensions by Dubinin (see Section 2) we can use Theorem 4.1 to generalise a classical symmetrisation theorem of Baernstein. We need to introduce some more notation: Let $\mathbb{B}$ be the unit ball in $\mathbb{R}^{n}, n \geqslant 2$ with centre at 0 . If $D$ is a domain with $D \subset \mathbb{B}$, we denote by $D^{\sharp}$ the spherical symmetrisation of $D$. The domain $D^{\sharp}$ is defined by the property: the intersection of $D^{\sharp}$ with every sphere $|x|=r, 0<r<1$ is a spherical cap centred on the $x_{1}$-axis with spherical measure equal to the spherical measure of the intersection of $D$ with the same sphere; for details see [2]. If $f \in L^{1}(\partial \mathbb{B})$, we denote by $f^{\sharp}$ the symmetric decreasing rearrangement of $f$ (see for example, [2, Section 2]).

THEOREM 5.1. Let $f \in L^{1}(\partial \mathbb{B}), f \geqslant 0$ and $f>0$ on a set of positive capacity. We denote by $h$ the harmonic extension of $f$ in $\mathbb{B}$ and by $h^{\sharp}$ the harmonic extension of $f^{\sharp}$ in $\mathbb{B}$. Let $D \subset \mathbb{B}$ be a domain with $h$-resolutive boundary $\partial D$ and assume that $\partial D^{\natural}$ is $h^{\natural}$-resolutive. Then for $E \subset \partial \mathbb{B}$,

$$
\mu_{D}^{h}(0, E) \leqslant \mu_{D^{\sharp}}^{h^{\sharp}}\left(0, E^{\sharp}\right),
$$

where $E^{\sharp}$ is the spherical cap on $\partial \mathbb{B}$ centred at the point $(1,0, \ldots, 0)$ and with spherical measure equal to the spherical measure of $E$.

For $f \equiv 1$ and $E=\partial \mathbb{B}$, this theorem was proved by Baernstein $[1](n=2)$ and by Baernstein and Taylor [2] $(n \geqslant 3)$ who used the star-function method. Another approach to this classical result (via polarisation) was used in [15] and [6]. This approach leads also from the polarisation Theorem 4.1 to the symmetrisation Theorem 5.1. For $n=2$, Theorem 5.1 implies an extension of the Beurling-Nevanlinna projection theorem [14, ppi08-110]. The precise statement is left to the reader. 


\section{CONCLUDING REMARKS AND OPEN PROBLEMS}

Bañuelos and Carroll [3, 4] formulated the statement that in a simply connected planar domain conditional Brownian paths tend to follow hyperbolic geodesics. This is a usefull general principle. Several results in [3] and in [12] agree with this principle. The results of the present paper also support it. However, it should be admitted that the above statement has only a heuristic and intuitive character, and that the known theorems do not give a satisfactory quantitative version of it.

ConJeCture 1 . Let $\gamma$ be an arc in a Jordan domain $D$ joining two points $\xi, \zeta \in D \cup \partial D$. For $\varepsilon>0$, let $D(\varepsilon, \gamma):=\{z \in D: \operatorname{dist}(z, \gamma)<\varepsilon\}$. Then the probability that a Brownian motion conditioned to travel from $\xi$ to $\zeta$ stays inside $D(\varepsilon, \gamma)$ is maximised when $\gamma$ is the hyperbolic geodesic of $D$ joining $\xi$ and $\zeta$.

To extend the Bañuelos-Carroll principle to more general contexts we need to abandon hyperbolic geodesics. In a simply connected domain the hyperbolic geodesics are exactly the Green lines (the orthogonal trajectories of the level curves of the Green function). So it is reasonable to conjecture that conditional Brownian paths in a Greenian domain in $\mathbb{R}^{n}$ (or even more generally in an abstract Greenian space) tend to follow the Green lines.

Despite recent progress the expected lifetime of conditional Brownian motion is not well-understood even in simply connected planar domains. Let $E_{a}^{b} T_{D}$ denote the expected lifetime of Brownian motion in $D$ conditioned to travel from $a$ to $b(a, b \in D \cup \partial D)$. Griffin, McConnell, and Verchota [12] proved that if $z, \zeta, \xi$ lie in this order on a hyperbolic geodesic of $D$ and if we assume that

(a) $D$ has finite area.

(b) $\xi \in \partial D$

then $E_{z}^{\xi} T_{D} \geqslant E_{\zeta}^{\xi} T_{D}$. They conjectured that each of the assumptions (a) and (b) is redundant. However, their ingenious methods depend in an essential way on these assumptions and their conjectures remain open.

Another beautiful result for the expected lifetime was proved in [5] and [12]: Let $\xi, \zeta$ be two points on the unit circle. The lifetime of Brownian motion conditioned to travel from $\xi$ to $\zeta$ is maximised when the points $\xi, \zeta$ are diametrically opposite. For the ball in $\mathbb{R}^{n}, n \geqslant 3$, an analogous statement is not known. A related conjecture is the following:

CONJECTURE 2 . Let $D$ be a simply connected planar domain, symmetric with respect to the real line. Let $\xi, \zeta \in D \cap \mathbb{R}_{+}^{2}$ Then $E_{\xi}^{\zeta} T_{D}<E_{\xi}^{\hat{\zeta}} T_{D}$.

Intuition suggests that if $\xi, \zeta$ are far away from each other then $E_{\xi}^{\zeta} T_{D}$ should be large. So $E_{\xi}^{\zeta} T_{D}$ provides a "measure" of the distance between the points $\xi$ and $\zeta$ relative to $D$.

CONJECTURE 3 . In a simply connected domain $D$ the function $d_{D}: D \times D \rightarrow \mathbb{R}$ defined by $d_{D}(\xi, \zeta)=E_{\xi}^{\zeta} T_{D}$ is a metric in $D$. 
Some other conjectures for conditional Brownian motion appear in [12] and [4]. One of them is the following: Among all convex domains of area equal to $\pi$, a unit disk has the smallest maximal expected lifetime of conditional Brownian motion. This maximal expected lifetime for the unit disk is obtained for Brownian motion conditioned to travel between antipodal points. In [12] it is computed to be equal to $4 \ln 2-2 \approx 0.77$. Let $Q$ be a square of area $\pi$, and let $\xi, \zeta$ be the end-points of a diagonal of $Q$. It is a nice and instructive exercise on elliptic functions to compute the expected lifetime of Brownian motion in $Q$ conditioned to travel from $\xi$ to $\zeta$. We found it to be approximately equal to 0.79 . So this computation supports the above conjecture. We note, however, that there is no general result which implies that the maximal lifetime for the square $Q$ is attained for the points $\xi, \zeta$. This fact illustrates once more our lack of understanding of the geometric behaviour of conditional Brownian motion.

NOTE ADDED ON 29-5-2002. The above conjecture has recently been disproved in [13]. I thank the referee for this information.

\section{REFERENCES}

[1] A. Baernstein II, 'Integral means, univalent functions and circular symmetrization', Acta Math. 133 (1974), 139-169.

[2] A. Baernstein II and B.A.Taylor, 'Spherical rearrangements, subharmonic functions, and *-functions in n-space', Duke Math. J. 43 (1976), 245-268.

[3] R. Bañuelos and T.Carroll, 'Conditioned Brownian motion and hyperbolic geodesics in simply connected domains', Michigan Math. J. 40 (1993), 321-332.

[4] R. Bañuelos and T. Carroll, 'Extremal problems for conditioned Brownian motion and the hyperbolic metric', Ann. Inst. Fourier (Grenoble) 50 (2000), 1507-1532.

[5] R. Bañuelos and E. Houswarth, 'An isoperimetric-type inequality for integrals of Green's functions', Michigan Math. J. 42 (1995), 603-611.

[6] D. Betsakos, 'Polarization, conformal invariants and Brownian motion', Ann. Acad. Sci. Fenn. Ser.A I Math. 23 (1998), 59-82.

[7] F. Brock and A. Yu. Solynin, 'An approach to symmetrization via polarization', Trans. Amer. Math. Soc. 352 (2000), 1759-1796.

[8] J.L. Doob, 'Conditional Brownian motion and the boundary limits of harmonic functions', Bull. Soc. Math. France 85 (1957), 431-458.

[9] J.L. Doob, Classical potential theory and its probabilistic counterpart, Fundamental Principles of Mathematical Sciences 262 (Springer-Verlag, New York, 1984).

[10] V.N. Dubinin, 'Capacities and geometric transformations of subsets in n-space', Geom. Funct. Anal. 3 (1993), 342-369.

[11] V.N. Dubinin, 'Symmetrization in the geometric theory of functions of a complex variable', Russian Math. Surveys 49 (1994), 1-79.

[12] P.S.Griffin, T.R.McConnell and G.C.Verchota, 'Conditioned Brownian motion in simply connected planar domains', Ann. Inst. H. Poincaré Probab. Statist. 29 (1993), 229-249.

[13] B. Kawohl and G. Sweers, 'Among all 2-dimensional convex domains the disk is not optional for the lifetime of a conditioned Brownian motion', J. Analyse Math. (to appear). 
[14] R. Nevanlinna, Analytic functions, (first edition in German 1936) (Springer-Verlag, New York, 1970).

[15] A. Yu. Solynin, 'Functional inequalities via polarization', (in Russian), Algebra i Analiz 8 (1996), 148-185; English translation in St.Petersburg Math. J. 8 (1997), 1015-1038.

[16] V. Wolontis, 'Properties of conformal invariants', Amer. J. Math. 74 (1952), 587-606.

Department of Applied Mathematics

University of Crete

71409 Heraklio

Greece

e-mail: betsakos@tem.uoc.gr
Current address:

Department of Mathematics

Aristotle University of Thessaloniki

54006 Thessaloniki

Greece 